\title{
BMJ Open Protocol of a multimethod descriptive study: adapting hospital-to-home transitional care interventions to the rural healthcare context in Ontario, Canada
}

\author{
Mary T Fox, ${ }^{\oplus 1,2}$ Souraya Sidani, ${ }^{\oplus}$ Jeffrey I Butler, ${ }^{1}$ Mark W Skinner, ${ }^{\oplus 4}$ \\ Manal Alzghoul ${ }^{\top 5}$
}

To cite: Fox MT, Sidani S, Butler Jl, et al. Protocol of a multimethod descriptive study: adapting hospital-to-home transitional care interventions to the rural healthcare context in Ontario, Canada. BMJ Open 2019;9:e028050. doi:10.1136/ bmjopen-2018-028050

- Prepublication history and additional material for this paper are available online. To view these files, please visit the journal online (http://dx.doi. org/10.1136/bmjopen-2018028050).

Received 19 November 2018 Revised 12 March 2019 Accepted 27 March 2019

Check for updates

(c) Author(s) (or their employer(s)) 2019. Re-use permitted under CC BY-NC. No commercial re-use. See rights and permissions. Published by BMJ.

For numbered affiliations see end of article.

Correspondence to

Dr Mary T Fox;

maryfox@yorku.ca

\section{ABSTRACT}

Introduction Faced with costly hospital readmissions of increasingly complex patient populations, transitional care is a priority throughout Ontario, Canada; yet, rural patients have significantly more hospital readmissions and emergency department visits during the first 30 days following hospitalisation than urban patients. Because transitional care (TC) was designed and evaluated with urban patients, addressing urban-rural disparities in TC effectiveness requires increasing the alignment of $\mathrm{TC}$ with the needs of patients and families in rural communities and the rural nursing practice context. The study objectives are to (1) determine the perceived acceptability of evidence-based TC interventions targeting postdischarge care management to patients, families and nurses and (2) adapt the interventions to patients' and families' needs and the rural nursing practice context.

Methods and analysis This multimethod study has two phases. In phase I, 32-48 patients and families will rate their level of preparedness for discharge and the acceptability of evidence-based TC interventions. Participants will be engaged in semi-structured interviews about their care management needs, their perspectives on the interventions in fitting those needs and in providing suggestions for adapting the interventions to fit their needs. TC interventions perceived as acceptable to patients and families will be examined in phase II. In phase II, 32-48 hospital and home care nurses will rate the acceptability of the interventions identified by patients and families and attend focus group discussions on the feasibility of providing the interventions. Phase I and II data will be analysed using descriptive statistics and qualitative content analysis.

Ethics and dissemination Ethics approval was obtained from the Research Ethics Board at York University and participating hospital sites. Findings will be communicated through plain language fact sheets, policy briefs, pressreleases and peer-reviewed conference presentations and manuscripts.

\section{INTRODUCTION}

Poorly planned and executed hospital discharges are associated with emergency

\section{Strengths and limitations of this study}

- This study uses a systematic process to assess the perspectives of patients, families and nurses on the acceptability of hospital-to-home transitional care interventions and, based on those perspectives, to adapt the interventions for rural transitional care.

- Assessing the acceptability of the interventions will highlight intervention aspects requiring modification to enhance intervention responsiveness, uptake, implementation and effectiveness.

- The study uses telephone interviews and focus groups to overcome the logistical and geographical barriers to conducting research in rural communities.

- The self-report measures are subject to response bias.

- The study is limited to Ontario, Canada and its findings may not be generalisable to other countries.

department visits and hospital readmissions, which are costly to the healthcare system. ${ }^{1}$ Hospital-to-home transitional care (TC) involves time-limited interventions delivered predominantly by nurses during and following hospitalisation that focus on continuity of care; supporting patients and families in managing postdischarge care is a hallmark of high-quality TC. ${ }^{2}$ With established benefits in improving outcomes, TC is a priority in Ontario, Canada. Yet, patients in rural communities have significantly higher emergency department visits and hospital readmissions than their urban counterparts. ${ }^{3}$ Almost $60 \%$ of rural patients visit the emergency department for a non-urgent health problem within 30 days of discharge, and up to $59 \%$ of their hospital readmissions-which are conservatively estimated to unnecessarily cost the health system \$C162 million/yearare preventable. ${ }^{3}$ These findings suggest 
inadequacies in preparing patients and families to manage postdischarge care, and they highlight the need to optimise TC in rural communities to resolve rural-urban TC inequities. However, four key gaps in knowledge and practice, validated in our prior stakeholder engagement activities, need to be addressed to optimise TC in rural communities. $^{4}$

\section{Non-alignment of TC with the rural context}

Rural communities have higher proportions of older people, higher rates of multiple chronic conditions ${ }^{5}$ and more people of lower socioeconomic status than urban communities, ${ }^{6}$ which are all risk factors for hospital readmission. ${ }^{3}$ Furthermore, rural communities are characterised by longstanding trends of health-service deprivation (less health system infrastructure and human health resources), and barriers to accessibility and utilisation related to distance and widely dispersed patient populations. ${ }^{67}$ These characteristics have been ignored in prior TC trials, which excluded rural communities, limiting the applicability of their findings.

\section{Lack of fit between the rural nursing practice context and TC research trials}

TC is mostly nurse-led and Ontario nurses report being responsible for providing TC. $^{28}$ Most TC is based on trials where patients had extensive support from Master's prepared Advanced Practice Nurses from hospital admission to up to 3 months after discharge, with frequent home visits. ${ }^{9-14}$ Although rural nurses practice with a high degree of autonomy, they do so with fewer resources, less education and across larger geographical regions than nurses in TC trials. ${ }^{15}$ When there is poor fit between provider, patient and setting characteristics of trials versus those of the practice area where the interventions are implemented, adaptation is extensive. ${ }^{16}$ These adaptations can result in considerable intervention implementation drift to the extent that the adapted interventions no longer resemble the original ones evaluated in the trials. ${ }^{16}$ Adapted interventions with substantial drift fail to improve outcomes, highlighting the need to work with nurses in applying a systematic process to intervention adaptation. ${ }^{17}$

\section{Limited functional care planning in TC}

People living with chronic conditions have poorer functional health and greater risk of hospital-related functional decline and disability. ${ }^{18} 19$ Functional decline and related complications (eg, falls) are key causes of hospital readmission in at-risk populations. ${ }^{20}$ Consequently, rural TC requires interventions that meet patients' functional needs; yet, few TC trials focus on functioning. ${ }^{21}{ }^{22} \mathrm{We}$ found only two TC trials focusing on functioning, but they, too, were conducted in urban communities, ${ }^{12} 21$ limiting generalisability of their findings. Furthermore, $40 \%$ and $76 \%$ of eligible trial patients, respectively, declined participation, ${ }^{121}$ raising doubts about the relevance of the interventions, and highlighting the need to better understand patients' perspectives on interventions that aim to optimise their functioning during the postdischarge recovery period.

\section{Non-alignment of TC with families' needs}

Because rural communities have fewer healthcare services, families are essential to effective TC. ${ }^{2}$ Many families are expected to perform care activities that, in hospital, were performed by nurses, and patients with multiple chronic conditions require more complex care management. ${ }^{23}$ The functional abilities and care requirements of these patients often differ substantially following hospital discharge than pre-admission, and most families feel unprepared to successfully manage postdischarge care- $67 \%$ of families report that their postdischarge caregiving needs are not addressed. ${ }^{2}$ These findings underscore the urgency of aligning TC with families' caregiving needs. Alignment enhances TC's responsiveness to families' needs and increases their caregiving confidence, ultimately improving patient and system outcomes. ${ }^{17}$

\section{Study objectives}

The study objectives are to (1) determine the perceived acceptability of evidence-based TC interventions to patients, families and nurses and (2) adapt the interventions to fit patients' and families' needs and the rural nursing practice context.

\section{Research questions}

The research questions include:

1. What are the perspectives of rural-dwelling patients and their families on the acceptability of the TC interventions in fitting their postdischarge care management needs?

a. How prepared are rural-dwelling patients and families to manage postdischarge care?

b. What are their unmet postdischarge care management needs?

c. Which TC interventions are acceptable, meet their needs/fail to meet their needs and why?

d. What suggestions do patients and families have for adapting TC interventions to improve fit with their needs?

2. How do hospital and home care nurses serving rural communities perceive the feasibility of the TC interventions identified by patients and families as acceptable and fitting their needs?

a. Which interventions are acceptable and feasible to nurses, and which should be modified and how?

b. What barriers and facilitators may influence their implementation?

c. What resources do nurses need to implement them?

\section{Transitional care interventions}

The evidence-based TC interventions (hereafter, referred to as interventions) are derived from prior reviews of studies in acute and postacute care settings. They target postdischarge care management needs and include: discharge planning, managing treatments (including 
Table 1 Description of transitional care interventions

\begin{tabular}{|c|c|c|c|c|}
\hline Aspect & Discharge planning & Treatments & Warning signs & Physical acitivity \\
\hline Goals & $\begin{array}{l}\text { Prepare patient and family to } \\
\text { manage care and recovery } \\
\text { at home after discharge; } \\
\text { continue to meet patient } \\
\text { needs after discharge; ensure } \\
\text { patient care is coordinated as } \\
\text { patient returns home. }\end{array}$ & $\begin{array}{l}\text { Ensure patient and family know } \\
\text { the treatments (eg, medications, } \\
\text { wound care) to be used/applied } \\
\text { at home; increase patient and } \\
\text { family confidence and ability } \\
\text { to use treatments correctly; } \\
\text { improve patient health. }\end{array}$ & $\begin{array}{l}\text { Ensure patient and family } \\
\text { know the warning signs that } \\
\text { indicate worsening health } \\
\text { conditions and what to do } \\
\text { about them. }\end{array}$ & $\begin{array}{l}\text { Ensure patient and family } \\
\text { understand the importance } \\
\text { of physical activity during } \\
\text { recovery; promote safe } \\
\text { physical activity; prevent } \\
\text { declines in patient ability } \\
\text { to perform daily physical } \\
\text { activities; promote patient } \\
\text { return to usual daily activities. }\end{array}$ \\
\hline $\begin{array}{l}\text { Key } \\
\text { components } \\
\text { and activities }\end{array}$ & $\begin{array}{l}\text { Assess: patient and family } \\
\text { needs related to managing } \\
\text { care and recovery at home. } \\
\text { Involve patient and family in: } \\
\text { setting goals and planning } \\
\text { care; identifying strategies to } \\
\text { meet goals and home care } \\
\text { needs. } \\
\text { Prepare patient and family } \\
\text { how to manage care } \\
\text { and recovery at home } \\
\text { after discharge (provide } \\
\text { instructions and demonstrate } \\
\text { skills). } \\
\text { Address barriers to meeting } \\
\text { goals. } \\
\text { Organise and coordinate care } \\
\text { with community providers. }\end{array}$ & $\begin{array}{l}\text { Assess: patient self-care/ } \\
\text { family care management and } \\
\text { learning needs; treatments } \\
\text { prescribed but not used } \\
\text { properly and underlying } \\
\text { reasons. } \\
\text { Involve patient and family } \\
\text { in: setting goals related to } \\
\text { managing treatments after } \\
\text { discharge and identifying } \\
\text { strategies to meet goals. } \\
\text { Teach patient and family about } \\
\text { treatments to be continued } \\
\text { at home, provide verbal } \\
\text { and written instructions, } \\
\text { demonstrate use or application } \\
\text { of treatments. } \\
\text { Resolve treatment } \\
\text { discrepancies based on } \\
\text { problem identified. } \\
\text { Ensure that medication } \\
\text { reconciliation has been } \\
\text { performed on hospital } \\
\text { admission and discharge and } \\
\text { on returning home. }\end{array}$ & $\begin{array}{l}\text { Assess: patient and family } \\
\text { learning needs about } \\
\text { potential warning signs } \\
\text { specific to patient's health } \\
\text { conditions or surgical } \\
\text { procedures. } \\
\text { Teach patient and family } \\
\text { about warning signs (eg, } \\
\text { explain, demonstrate and/or } \\
\text { provide written information } \\
\text { on how to monitor and } \\
\text { recognise them, what to do, } \\
\text { when to get medical help, } \\
\text { who to contact and when to } \\
\text { go to the emergency room). }\end{array}$ & $\begin{array}{l}\text { Assess: patient level of } \\
\text { mobility and need for assistive } \\
\text { devices; patient and family } \\
\text { physical activity learning } \\
\text { needs; barriers to physical } \\
\text { activity. } \\
\text { Involve patient and family in } \\
\text { setting physical activity goals } \\
\text { and identifying strategies to } \\
\text { meet those goals. } \\
\text { Teach patient and family about } \\
\text { risks of bed rest, safe mobility, } \\
\text { balancing rest with physical } \\
\text { activity. } \\
\text { Increase physical activity } \\
\text { based on patient goals, } \\
\text { physical abilities, activity } \\
\text { tolerance, and health } \\
\text { conditions. } \\
\text { Address barriers to performing } \\
\text { physical activity. }\end{array}$ \\
\hline
\end{tabular}

All interventions are initiated within 24 hours of hospital admission and continued daily throughout hospital stay. Discharge planning lays the foundation for the other three interventions and ends at hospital discharge. The other interventions continue after discharge for 1 month, with home visit and telephone follow-up, and their continued need is reassessed at 1 month postdischarge.

medications), identifying and responding to warning signs and restoring physical functioning. ${ }^{12224-28}$ The four interventions are summarised in table 1.

\section{METHODS}

\section{Study design}

This multimethod, two-phase, descriptive study (figure 1) is guided by the Intervention Acceptability and Collaborative Intervention Planning Frameworks, which combine a patient-centred approach to intervention adaptation and the principles of community-based research, and makes engaging healthcare consumers (eg, patients and families) and providers (eg, nurses) in adaptation an explicit priority. ${ }^{17}{ }^{29}$ Adaptation involves modifying or revising aspects of an intervention (eg, delivery method) based on consumers' and providers' perspectives of the interventions. ${ }^{29}$ The framework provides direction on systematically adapting interventions to align them with consumers' needs in particular healthcare contexts, while preserving the interventions' essential elements, to enhance intervention responsiveness, uptake, implementation and, ultimately, effectiveness.

Two phases of activities are planned for this study. In phase I, TC consumers (patients and families) will be engaged in assessing the interventions' acceptability in meeting their postdischarge care management needs and inform adaptation of the interventions to fit these needs. In phase II, TC providers (nurses) will be engaged in assessing the interventions' acceptability and feasibility for the rural nursing practice context and inform adaptation to this context. Both phases use logic models (tailored to the participant group targeted in each phase) to promote understanding of the interventions (online supplementary material, appendix A). Logic models contain information, synthesised from empirical literature, depicting an intervention's (1) goals (what it aims to achieve); (2) 
PHASE I:

Transitional Care Consumers

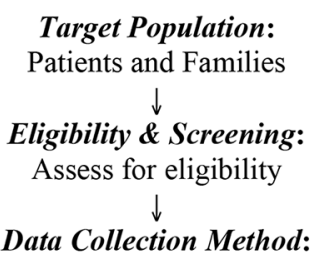

Telephone interviews to administer measures and ask semi-structured questions $\downarrow$

Focus on:

Transitional care experience and unmet needs

Preparedness for managing post-discharge care

Perceived acceptability of 4 transitional care interventions

Suggestions for modifying interventions to meet patients' and families' needs

$$
\downarrow
$$

Findings:

Identification of interventions viewed as acceptable and of modifications to make $\downarrow$

Outputs:

Acceptable and modified transitional care interventions moved to next phase

\begin{tabular}{c}
\hline PHASE II: \\
Transitional Care Providers \\
\hline Target Population: \\
Nurses \\
$\downarrow$ \\
Eligibility $\&$ Screening: \\
Assess for eligibility \\
$\downarrow$
\end{tabular}

Data Collection Method:

Self-administration of a measure of each interventions' acceptability and telephone semistructured focus group interviews

$$
\text { Focus on: }
$$

Perceived acceptability of transitional care interventions identified as acceptable to patients and families

Potential facilitators and barriers to implementing the transitional care interventions Resources needed to deliver the transitional care interventions

Suggestions for modifying interventions to enhance feasibility of their implementation in the rural practice context

\section{Findings:}

Identification of interventions viewed as acceptable and feasible

$$
\downarrow
$$

Outputs:

Acceptable and feasible transitional care interventions - evaluation in future trial of outcomes in rural practice context

Figure 1 Study flow diagram.

processes involving its components and activities (what it entails), mode of delivery (how it is given), the human and material resources for providing it, dose (eg, how many sessions, how often) and (3) outcomes (the shortterm and long-term outcomes that are expected). ${ }^{30} 31$ Both phases will be conducted in collaboration with two hospitals that serve different rural regions of Ontario (Southwestern and Northeastern) with data collection beginning in Summer 2018 and continuing through Spring, 2019.
Phase I: patient and family perspectives Sample and setting

The target population is rural-dwelling adults, at risk for readmission and the families of these patients. The purposeful sample will be stratified by role (patient or family), hospital region (Southwestern and Northeastern Ontario) and includes English-speaking and reading adults, aged 18 years and older. Eligible patients: are being discharged from hospital for a medical condition or surgical procedure-these patients have the highest 
readmission rates $(65 \%$ and $13.3 \%$, respectively $)$ in rural Ontario, live in a region with a Rurality Index for Ontario $>40,{ }^{32}$ are at risk for readmission (indicated by a LACE score $\geq 8)^{33}$ and are cognitively able to respond to questions (indicated by a Mini-Mental State Examination (MMSE) score $\geq 24$ ). ${ }^{34}$ Patients will be excluded if they are: admitted for palliation; unable to stand or weightbear at discharge (evidenced by a score of 0 on the corresponding item of the Basic Physical Capability Scale), ${ }^{35}$ or transferred to a rehabilitation, long-term or alternate level of care prior to returning home which would preclude their consideration of the interventions in the hospital-to-home postdischarge period. Eligible families are unpaid, primary care partners (eg, relatives, partners or friends) ${ }^{2}$ of patients meeting all eligibility criteria, with the exception of the cognitive ability criterion. Between 8-12 patients and 8-12 families will be interviewed per region (for a total of 32-48 TC consumers), depending on informational saturation within each region. ${ }^{36}$ This sample size was determined based on recommendations that 12-60 participants, with an average of 30, are needed to achieve saturation in qualitative studies ${ }^{37}$; this sample size is also sufficient for the study's quantitative descriptive purposes. ${ }^{38}$

\section{Variables and measures}

\section{Screening measures}

Patients' cognitive ability to respond to questions will be determined with the MMSE score documented in their medical record; the MMSE has established reliability and validity. ${ }^{34}$ Inability to stand or weight-bear will be measured by self-report using the corresponding item of the Basic Physical Capability Scale ${ }^{35}$; the scale demonstrated construct validity (all items fit the Rasch model testing) and internal consistency reliability (Cronbach's $\alpha=0.79$ ) in acute and long-term care samples. ${ }^{35}$ Data on patients' palliation and transfer status will be extracted from the patients' medical record using a standard form.

The rurality of the area where patients live will be measured by the Rurality Index for Ontario. ${ }^{32}$ The index is a census-based metric, derived from population size and density and travel times to nearest basic and advanced referral centres, developed for rural healthcare planning and policy development purposes. ${ }^{39}$ Scores are obtained by asking the patient or family for the patient's postal code and entering it into an online calculator; a score $\geq 40$ is considered rural ${ }^{40}$ and higher scores indicate higher degrees of rurality. ${ }^{32}$

Patients' risk for hospital readmission will be measured by the LACE index (through extraction from the medical record) ${ }^{33}$ The index uses four variables to predict patients' risk for hospital readmission during the 30-day discharge period: length of hospital stay (' $L$ '), acuity of the hospital admission ('A'), comorbidities ('C') and emergency department visits in the 6 months before admission (E) ${ }^{41}$ The LACE index was validated using administrative data from 1000000 patients and the four variables were significantly associated with 30-day hospital readmission. ${ }^{41}$ The index demonstrated discriminant ability in the hospital readmission of 4812 medical and surgical patients discharged from 11 Ontario hospitals (C statistic $=0.68,95 \%$ CI 0.65 to 0.71 ); a one-point increase in the index was associated with an $18 \%$ increase in 30-day hospital readmission (OR 1.18, 95\% CI 1.14 to 1.21$).{ }^{41}$ Family screening variables (eg, a primary care partner) will be measured by self-report using a standard form.

Preparedness for managing postdischarge care

Patients' and families' preparedness for managing postdischarge care will be measured by the Care Transition Measure, a 15-item self-report measure with 4 response options from strongly disagree (1) to strongly agree (4). The total score is derived by taking the mean of the items' scores; higher scores reflect a higher level of preparedness for discharge. The measure was content validated with 49 discharged patients and their families, and demonstrated construct validity (item correlations ranged from 0.25 to 0.75 with the items of a conceptually related tool), ${ }^{42}$ discriminant validity (discriminated between patients with postdischarge emergency visits and rehospitalisations) and internal consistency reliability (Cronbach's $\alpha=0.93){ }^{43}$

\section{Perceived intervention acceptability}

Patients' and families' perceived intervention acceptability will be measured by the self-report Intervention Acceptability Scale. The scale provides a written description of each intervention followed by a set of items to rate its acceptability in terms of its appropriateness, effectiveness, side effects/risks and ease of use. A 5-point scale ranging from not at all (0) to very much (4) is used in the rating. The total score is derived by taking the mean of the items' scores. The scale has demonstrated internal consistency reliability $(\alpha>0.80)$ and factorial validity (factor loadings $>0.30)^{44}$

\section{Sociodemographic and health characteristics}

Patients' sociodemographic and health characteristics will be measured using standard self-report questions on age, gender, marital status, employment status, occupation, living arrangement, highest level of education, ethnicity and number of days since hospital discharge; reason for hospital admission will be extracted from the medical record. Families will respond to similar standard questions to describe their own sociodemographic characteristics, in addition to their caregiving relationship (eg, spousal caregiver).

\section{Data collection procedures}

The data collection materials and procedures will be pilot tested for clarity, comprehension and time commitment prior to use in the full study. Potential eligible participants will be identified by hospital nurses who will introduce the study, and ask participants for permission to give their names to the Research Associate (RA). The RA will contact interested participants to explain the study, obtain consent, confirm eligibility using the screening measures (as described above) and arrange the interviews. 
To overcome logistical and geographic barriers characteristic of rural communities, consenting participants will be interviewed by telephone within 30 days of hospital discharge. Telephone interviews, which we have used previously, are less intimidating and as effective as faceto-face interviews, ${ }^{45}$ and will be conducted by the RA. Prior to the interview, the RA will mail interview materials including the logic models describing the interventions; the Care Transitions Measure assessing preparedness for managing postdischarge care; the Intervention Acceptability Scale assessing intervention acceptability and sociodemographic and health questions assessing participants' characteristics.

Participants will be asked to remove distractions from the environment such as turning off the TV or radio and to go to a quiet area for the interview to optimise communication. Participants will be asked to follow the measures to promote understanding. The RA will first administer the Care Transitions Measure, and then ask semi-structured questions about participants' TC experiences to identify needs. The RA will then describe each intervention using the information in the respective logic model and ask participants to rate each intervention's acceptability in addressing their needs. This will be followed by semi-structured questions (eg, What part(s) of this intervention can be changed to better address your needs?) focusing on how the interventions fit/do not fit their needs, intervention aspects that should be modified and how. ${ }^{46}$ Interviews will be $60-90 \mathrm{~min}$, audio-recorded and transcribed.

\section{Data analysis}

Descriptive statistics, commensurate with each variable's level of measurement, will be used to describe participants' average standing on preparedness for managing postdischarge care, perceived acceptability of the interventions and demographic variables. Acceptable interventions have a mean rating $>2$ (ie, midpoint of the response scale) and qualitative comments indicate that they fit participants' needs-even if adaptations are required. ${ }^{44}$ Conventional qualitative content analysis of the interview transcripts will be conducted concurrently with data collection (facilitated by NVivo 11) and will focus on identifying the postdischarge care management needs of patients and families and the interventions they perceive as acceptable/unacceptable and fitting/not fitting their needs. ${ }^{48}$ Analysis will involve developing preliminary codes and, through an iterative process, synthesising them into meaningful, hierarchically organised categories and subcategories. ${ }^{48}$ Definitions will be developed for each code, category and subcategory and their interconnections documented; exemplars for each will be identified. We will examine data for trends in participants' narratives by their scores on the Care Transitions Measure, Rurality Index for Ontario and Intervention Acceptability Scale using role-ordered and conceptually clustered matrices ${ }^{48}$ and analytic memoing. ${ }^{49}$ Strategies for trustworthiness will be employed (eg, confirmability by documenting an audit trail; credibility through independent data analysis by members of the research team; dependability through in-depth methodological description and transferability through the provision of data on participant and site characteristics).$^{50}$ Findings will enable the identification of interventions that fit patients' and families' needs and aspects requiring adaptation, and inform revision of the interventions. Our research team will review the findings and integrate patients' and families' feedback into the intervention logic models, which will be used in phase II of the study.

\section{Phase II: nurse perspectives}

\section{Sample and setting}

The purposive sample of nurses is stratified by their employment location (hospital vs home care) and hospital region (Southwestern vs Northeastern Ontario). Eligible hospital and home care nurses (eg, staff nurses, discharge planners) work at least 21 hours/week. Casual, temporary or agency nurses are ineligible. Separate, small $(n=4-6)$ telephone focus groups will be held with nurses from each stratum to maintain within-group homogeneity. Two focus groups will be held per stratum to ensure that a diversity of perspectives is captured, ${ }^{45}$ for a total of 8 focus groups with 32-48 TC providers, based on the same rationale as the estimated sample for phase I. Recruitment strategies include presenting the study at staff meetings and through email, and posting flyers with our contact information at the sites and on their social media accounts. Enrolment promoting strategies include conducting focus groups during non-working hours and providing a modest honorarium.

\section{Variables and measures}

Screening measures: Nurse screening variables (eg, hours worked per week) will be measured by self-report using a standard form. Perceived intervention acceptability: nurses' perceived intervention acceptability will be measured by the Intervention Acceptability Scale as previously described in phase I. Sociodemographic characteristics: nurses' sociodemographic characteristics will be measured with standard questions on age, gender, highest level of education, ethnicity in addition to employment role (eg, staff nurse, discharge planner).

\section{Data collection and procedures}

Nurses interested in participating in the study will contact the RA who will determine their eligibility, explain the study and obtain consent. Consenting nurses will be invited to focus groups to adapt the TC interventions, recommended by patients and families in phase $\mathrm{I}$, to the rural nursing context. Prior to the focus group sessions, participants will receive an interview package containing the intervention logic models, the Intervention Acceptability Scale, which nurses will have the option of completing using an online tool or in hard copy, to rate the acceptability of each intervention and a sociodemographic questionnaire. Our research team will review the 
nurses' scores from the Intervention Acceptability Scale and finalise the semi-structured interview guide. In the focus group sessions, the RA will use the logic models to describe the interventions and use the interview guide to engage nurses in discussing the feasibility of providing the interventions. Questions will prompt nurses to discuss how the interventions should be further adapted, while maintaining their essential elements and the adaptations recommended by patients and families in phase I, and considering the availability of resources to deliver the interventions. Examples include: 'What would help you deliver the interventions? How should this intervention be changed so that you can provide it in rural communities?' Focus groups sessions will be up to $90 \mathrm{~min}$, audio-recorded and transcribed.

\section{Data analysis}

Descriptive statistics, commensurate with each variable's level of measurement, will be used to describe nurses' acceptability of the interventions and sociodemographic characteristics. Transcripts of the focus group discussions will be content analysed ${ }^{47} 48$ (facilitated by NVivo 11) concurrently with data collection using qualitative analysis methods and strategies for trustworthiness, as described in phase I. We will examine data for trends in participants' narratives by their scores on the Intervention Acceptability Scale using role-ordered and conceptually clustered matrices, ${ }^{48}$ and analytic memoing. ${ }^{49}$ The perspectives of nurses will be summarised within each stratum and compared across strata (hospital vs homecare, Southwestern vs Northeastern Ontario) to identify patterns of similarity and difference. This context-sensitive approach will: 1) illuminate if and how practice location and region shape perceptions of the acceptability and feasibility of the interventions, barriers and facilitators in their future implementation, and the resources required to provide them and 2) enable the identification of commonalities in perceptions across regions that are likely to be transferable. Findings will enable the identification of intervention aspects requiring modification, and resources required by nurses to deliver the interventions in rural communities. Guided by these findings, we will revise the intervention logic models, and recommend resources to implement the interventions in rural communities.

\section{Patient and public involvement}

Members of the public informed the development of the research idea via telephone and teleconference engagement activities conducted in 2015. This resulting project includes a patient and family representative, a nurse and two healthcare administrators from rural Ontario. They have played essential roles by sharing their experiences to highlight areas of TC that require attention, which we have integrated into the project objectives and research plan. As the research project unfolds, they will advise on the conduct of the proposed activities and help to interpret the findings. They will assist in disseminating the findings by helping to tailor messages to members of the public.

\section{Ethics and dissemination}

Written informed consent will be obtained from all participants. All identifying information will be anonymised using alpha-numeric identifiers and pseudonyms created by a random name generator. All data will be stored on dedicated, encrypted, password-protected drives.

Findings will be communicated to relevant audiences through plain language fact sheets and policy briefs that will be tailored to end-users. These will be posted on the York University Centre for Aging Research and Education website. Findings will also be disseminated in a press-release and peer-reviewed journals.

\section{DISCUSSION}

Ontario's Bringing Care Home report highlights the need for robust evidence to inform patient-centred and family-centred TC practice and policy. ${ }^{52}$ This study will provide this evidence by improving understanding of patients' and families' hospital-to-home TC needs in rural Ontario. This understanding is fundamental to informing evidence-based TC policy development to address ruralurban disparities in TC effectiveness. ${ }^{53}$

Three potential benefits of the study's findings are anticipated. The first potential benefit is to improve the ability of rural patients, at risk for readmission following hospitalisation for medical conditions and surgical procedures and their families to manage postdischarge care. Evidence-based TC interventions, aligned to their postdischarge care management needs, will improve TC's responsiveness, which in turn will improve the ability of patients and families to manage postdischarge care, ultimately preventing unnecessary emergency department visits and hospital readmissions. Also, logic models describing these adapted interventions will be developed. These are useful tools to help patients and families understand how the TC interventions work, the activities they are involved in and how, knowledge of what to expect and enhance their ability to identify their needs.

The second potential benefit is to increase the capacity of nurses providing TC to rural patients at risk for hospital readmission and their families to incorporate the adapted TC interventions into their practice. Applying a systematic process in adapting TC interventions to the rural nursing practice context will enhance intervention feasibility and reduce the likelihood of individual nurse intervention adaptation as well as intervention implementation drift. TC interventions, aligned to the rural nursing practice context, will be more applicable, and more likely to be provided. Logic models describing how the interventions work, the activities that nurses need to perform, the time frames for their provision and the requisite resources will facilitate common understanding of what nurses should do to better meet the postdischarge care management needs of patients and families in rural communities. 
The third potential benefit is to influence TC policy. Administrators and policy-makers can use the findings to inform the development of TC policy for rural patients at risk for hospital readmission and their families.

Despite these potential benefits of the design, the study is limited to Ontario, Canada and its findings may not be generalisable to other countries. The self-report measures may be subject to response bias.

\section{Author affiliations}

${ }^{1}$ School of Nursing, York University, Toronto, Ontario, Canada

${ }^{2}$ York University Centre for Aging Research and Education, York University, Toronto, Ontario, Canada

${ }^{3}$ Daphne Cockwell School of Nursing, Ryerson University, Toronto, Ontario, Canada ${ }^{4}$ School of the Environment, Trent University, Peterborough, Ontario, Canada

${ }^{5}$ School of Nursing, Lakehead University, Thunder Bay, Ontario, Canada

\section{Acknowledgements The authors would like to thank the peer-reviewers.}

Contributors All authors made substantial contributions to the conception or design of the protocol, participated in drafting the protocol and revising it critically for important intellectual content. All authors read and approved the final version. In particular, MTF and SS conceived the study. MTF, SS, JIB and MWS designed it. MA helped design the semi-structured interview and focus-group guides. MTF, SS, JIB and MWS led the overall writing and drafting of the protocol. MA critically revised the protocol for important intellectual content.

Funding This work is supported by the Ontario Ministry of Health and Long-Term Care, Health System Research Fund, Grant No. 484.

Disclaimer The Ontario Ministry of Health and Long-Term Care had no role in the design of the protocol or in the writing of this manuscript.

Competing interests None declared.

Patient consent for publication Not required.

Ethics approval Ethics approval was obtained from the Research Ethics Board at York University (certificate \#: e2018-14) and participating hospital sites.

Provenance and peer review Not commissioned; externally peer reviewed.

Author note The postpilot test interview guides and measures are available from the first author on request.

Open access This is an open access article distributed in accordance with the Creative Commons Attribution Non Commercial (CC BY-NC 4.0) license, which permits others to distribute, remix, adapt, build upon this work non-commercially, and license their derivative works on different terms, provided the original work is properly cited, appropriate credit is given, any changes made indicated, and the use is non-commercial. See: http://creativecommons.org/licenses/by-nc/4.0/.

\section{REFERENCES}

1. Ray CA, Ingram V, Cohen-Mansfield J. Systematic review of planned care transitions for persons with dementia. Neurodegener Dis Manag 2015;5:317-31.

2. Gibson MJ, Kelly KA, Kaplan AK. Family caregiving and transitional care: a critical review [Internet]. Family Care Giver Alliance, National Centre on Care Giving [cited 2018 November]. https://www.caregiver. org/family-caregiving-and-transitional-care-critical-review-2012

3. Canadian Institute for Health Information. All-cause readmission to acute care and return to the emergency department. 2012 https:// secure.cihi.ca/estore/productFamily.htm?locale=en\&pf=PFC1823 (cited 2018 November).

4. Fox MT. Improving outcomes for older adults during acute care transitions in rural and suburban communities. Unpublished Report. Toronto: Canadian Institutes of Health Research, 2015.

5. Roberts KC, Rao DP, Bennett TL, et al. Prevalence and patterns of chronic disease multimorbidity and associated determinants in Canada. Health Promot Chronic Dis Prev Can 2015;35:87-94.

6. Skinner M, Hanlon N. Ageing resource communities: new frontiers of rural population change, community development, and voluntarism. London: Routledge, 2016.

7. Herron R, Skinner M, eds. Rural spaces and places of health and health care: handbook of health geography. London: Routledge international handbook series, 2018.
8. Fox MT, Butler JI. Nurses' perspectives on how operational leaders influence function-focused care for hospitalised older people. J Nurs Manag 2016;24:1119-29.

9. Lin PC, Wang CH, Chen CS, et al. To evaluate the effectiveness of a discharge-planning programme for hip fracture patients. J Clin Nurs 2009;18:1632-9.

10. Naylor MD, Brooten D, Campbell R, et al. Comprehensive discharge planning and home follow-up of hospitalized elders. JAMA 1999;281:613-20.

11. Naylor MD, Brooten DA, Campbell RL, et al. Transitional care of older adults hospitalized with heart failure: a randomized, controlled trial. $J$ Am Geriatr Soc 2004;52:675-84.

12. Naylor MD, Hirschman KB, Hanlon AL, et al. Comparison of evidence-based interventions on outcomes of hospitalized, cognitively impaired older adults. J Comp Eff Res 2014;3:245-57.

13. Rich MW, Beckham V, Wittenberg C, et al. A multidisciplinary intervention to prevent the readmission of elderly patients with congestive heart failure. N Engl J Med 1995;333:1190-5.

14. Rich MW, Vinson JM, Sperry JC, et al. Prevention of readmission in elderly patients with congestive heart failure: results of a prospective, randomized pilot study. J Gen Intern Med 1993;8:585-90.

15. Pitblado R. Characteristics and distribution of the regulated nursing workforce in rural and small town canada, 2003 and 2010: Prince George: Nursing practice in rural and remote Canada II, 2013:88.

16. Aarons GA, Green AE, Palinkas LA, et al. Dynamic adaptation process to implement an evidence-based child maltreatment intervention. Implement Sci 2012;7:1-9.

17. Cabassa LJ, Gomes AP, Meyreles Q, et al. Using the collaborative intervention planning framework to adapt a health-care manager intervention to a new population and provider group to improve the health of people with serious mental illness. Implementation Science 2014;9:1-11.

18. Kulig JC, Williams AM. Health in rural Canada. British Columbia: UBC Press, c2011.

19. Boyd CM, Landefeld CS, Counsell SR, et al. Recovery of activities of daily living in older adults after hospitalization for acute medical illness. J Am Geriatr Soc 2008;56:2171-9.

20. Slatyer S, Toye C, Popescu A, et al. Early re-presentation to hospital after discharge from an acute medical unit: perspectives of older patients, their family caregivers and health professionals. J Clin Nurs 2013;22:445-55.

21. Boltz M, Chippendale T, Resnick B, et al. Testing family-centered, function-focused care in hospitalized persons with dementia. Neurodegener Dis Manag 2015;5:203-15.

22. Fox MT, Persaud M, Maimets I, et al. Effectiveness of early discharge planning in acutely ill or injured hospitalized older adults: a systematic review and meta-analysis. BMC Geriatr 2013;13:70.

23. Kenning C, Coventry PA, Bower P. Self-management interventions in patients with long-term conditions: a structured review of approaches to reporting inclusion, assessment, and outcomes in multimorbidity. J Comorb 2014;4:37-45.

24. Coleman EA, Smith JD, Frank JC, et al. Preparing patients and caregivers to participate in care delivered across settings: the Care Transitions Intervention. J Am Geriatr Soc 2004;52:1817-25.

25. Fox MT, Sidani S, Persaud M, et al. Acute care for elders components of acute geriatric unit care: systematic descriptive review. J Am Geriatr Soc 2013;61:939-46.

26. Kind AJH, Brenny-Fitzpatrick M, Leahy-Gross K, et al. Harnessing Protocolized Adaptation in Dissemination: Successful Implementation and Sustainment of the Veterans Affairs Coordinated-Transitional Care Program in a Non-Veterans Affairs Hospital. J Am Geriatr Soc 2016;64:409-16.

27. Resnick B, Galik E, Boltz M. Function focused care approaches: literature review of progress and future possibilities. J Am Med Dir Assoc 2013;14:313-8.

28. Hesselink G, Schoonhoven L, Barach P, et al. Improving patient handovers from hospital to primary care: a systematic review. Ann Intern Med 2012;157:417-28.

29. Sekhon M, Cartwright M, Francis JJ. Acceptability of healthcare interventions: an overview of reviews and development of a theoretical framework. BMC Health Serv Res 2017;17:1-13.

30. Alkin MC, Vo AT, Hansen M. Using logic models to facilitate comparisons of evaluation theory. Eval Program Plann 2013;38:33.

31. Ball L, Ball D, Leveritt $M$, et al. Using logic models to enhance the methodological quality of primary health-care interventions: guidance from an intervention to promote nutrition care by general practitioners and practice nurses. Aust $J$ Prim Health 2017;23:53-60.

32. Ontario Medical Association. The rurality index for ontario (RIO) postal code look- up; 2017. [cited 2018 November] [about screen 1]. https://apps.oma.org/RIO/index.html 
33. Gruneir A, Dhalla IA, van Walraven C, et al. Unplanned readmissions after hospital discharge among patients identified as being at high risk for readmission using a validated predictive algorithm. Open Med 2011;5:104-11.

34. Crum RM, Anthony JC, Bassett S, et al. Population-based norms for the Mini-Mental State Examination by age and educational level. JAMA 1993;269:2386-91.

35. Resnick B, Galik E, Boltz M. Basic physical capability scale: psychometric testing with cognitively impaired older adults. Am J Alzheimers Dis Other Demen 2014;29:326-32.

36. Morse JM, Field PA. Qualitative research methods for health professionals. 2nd edn. Thousand Oaks: Sage, 1995.

37. Baker SE, Edwards R. How many qualitative interviews is enough? Expert voices and early career reflections on sampling and cases in qualitative research. NCRM Methods Review, 2012:1-43. http:// eprints.brighton.ac.uk/11632/1/how_many_interviews.pdf

38. Norman GR, Streiner DL. Biostatistics: the bare essentials. 4th ed. Canada, Hamilton, Ontario: B.C. Decker Inc, 2013.

39. Kralj B. Measuring 'rurality' for the purpose of health-care planning: an empirical measure for Ontario. OMR 2000;67:33-52.

40. Glazier RH, Zagorski BM, Rayner J. Comparison of primary care models in Ontario by demographics, case mix and emergency department use, 2008/09 to 2009/10. [Internet]. Institute for Clinicial Evaluative Sciences. [cited 2018 November]. https://www.ices.on. ca/Publications/Atlases-and-Reports/2012/Comparison-of-PrimaryCare-Models

41. van Walraven C, Dhalla IA, Bell C, et al. Derivation and validation of an index to predict early death or unplanned readmission after discharge from hospital to the community. CMAJ 2010;182:551-7.
42. Coleman EA, Smith JD, Frank JC, et al. Development and testing of a measure designed to assess the quality of care transitions. Int $J$ Integr Care 2002;2.

43. Coleman EA, Maloney E, Parry C. Assessing the quality of preparation for posthospital care from the patient's perspective: the care transitions measure. Med Care 2005;3:246-55.

44. Sidani S, Epstein DR, Bootzin RR, et al. Assessment of preferences for treatment: validation of a measure. Res Nurs Health 2009;32:419-31.

45. Krueger RA, Casey MA. Focus groups: a practical guide for applied research. 5th edn. Thousand Oaks: Sage, 2014.

46. Sidani S. Health intervention research: advances in research design and methods. London: Sage, 2015.

47. Hsieh HF, Shannon SE. Three approaches to qualitative content analysis. Qual Health Res 2005;15:1277-88.

48. Miles MB, Huberman AM, Saldana J. Qualitative data analysis. Los Angeles: Sage, 2013.

49. Bodgewic S. Participant observation. In: Miller BCW, ed. Doing qualitative research. Thousand Oaks: Sage, 1999:47-70.

50. Lincoln YS, Guba EG, Pilotta JJ. Naturalistic inquiry. New York: Sage, 1985:438-9.

51. Shenton AK. Strategies for ensuring trustworthiness in qualitative research projects. Education for Information 2004;22:63-75.

52. Donner G. Bringing care home. Report of the expert group on home and community care. [Internet] Expert Group on Home and Community Care. [cited 2018 November]. http://health.gov.on.ca/en/ public/programs/ccac/docs/hcc_report.pdf

53. Adopting a common approach to transitional care planning: helping health links improve transitional and coordination of care. [Internet]. Health Quality Ontario. [cited 2018 November]. https://Itctoolkit.rnao. ca/clinical-topics/care-transitions 